\title{
Nueva oferta educativa universitaria con enfoque intercultural: el caso de la Maestría en Lengua y Cultura Nahua de la Universidad Veracruzana ${ }^{1}$
}

Bernal-Lorenzo, Daisy; Figueroa-Saavedra, Miguel

Nueva oferta educativa universitaria con enfoque intercultural: el caso de la Maestría en Lengua y Cultura Nahua de la Universidad Veracruzana ${ }^{1}$

Revista Educación, vol. 43, núm. 2, 2019

Universidad de Costa Rica, Costa Rica

Disponible en: http://www.redalyc. org/articulo.oa?id=44058158001

DOI: https://doi.org/10.15517/revedu.v43i2.32934

Esta obra está bajo una Licencia Creative Commons Atribución-NoComercial-SinDerivar 3.0 Internacional. 


\title{
Nueva oferta educativa universitaria con enfoque intercultural: el caso de la Maestría en Lengua y Cultura Nahua de la Universidad Veracruzana ${ }^{1}$
}

\begin{abstract}
A new postgraduate program in intercultural-studies: the Masters Program in Nahua Indigenous Culture and Language at the University of Veracruz, Mexico ${ }^{2}$
\end{abstract}

Daisy Bernal-Lorenzo

Universidad Veracruzana, México

areanorling@gmail.com

http://orcid.org/0000-0001-9885-0999

Miguel Figueroa-Saavedra

Universidad Veracruzana, México

migfigueroa@uv.mx

D http://orcid.org/0000-0001-5990-1258
DOI: https://doi.org/10.15517/revedu.v43i2.32934 Redalyc: http://www.redalyc.org/articulo.oa?id=44058158001
Recepción: 15 Septiembre 2018

Aprobación: 28 Febrero 2019

\section{REsumen:}

El presente artículo tiene como objetivo demostrar, a través de una experiencia de diseño curricular de una oferta educativa con enfoque intercultural la necesidad y posibilidad de diseñar programas impartidos en una lengua indígena nacional (náhuatl) que contribuyan al respeto y valoración de la diversidad lingüística. Este diseño se hizo a partir de un diagnóstico de necesidades formativas, realizado entre 2015 y 2017 a través de cuestionarios a 67 profesionistas hablantes de LIN de Veracruz y 15 empleadores y autoempleados, y entrevistas enfocadas a 14 personas especialistas en el campo de la lingüística y la educación de instituciones veracruzanas, integrando y analizando los datos un equipo base de siete docentes-investigadores. Esta iniciativa se muestra como un ejemplo de buenas prácticas educativas en la demostración de la factibilidad de propuestas de planificación lingüística e intelectualización que doten de prestigio y estatus en la educación superior a lenguas minorizadas y discriminadas, y en el combate contra los prejuicios lingüísticos que aún rigen el sistema educativo mexicano, consolidando un modelo de educación intercultural en México.

Palabras clave: Política lingüística, Educación intercultural, Lengua indígena, Plan de estudios universitarios, Educación Superior.

\section{ABSTRACT:}

This objective of this article is to demonstrate that it is necessary and possible to design intercultural-based programs taught in Nahuatl, a native Mexican indigenous language, in order to preserve the country's heritage and indigenous linguistic diversity. This effort was based on an educational needs assessment carried out between 2015 and 2017 among sixty-seven professionals, fifteen employers and self-employed individuals as well as interviews with fourteen indigenous language and culture experts. The information gathered was organized by a group of seven faculty researchers. This initiative exemplifies good educational practices, since it shows the feasibility in proposing language and cultural studies for minoritized languages in higher education to oppose linguistic preconceptions within the Mexican Educational System and strengthen Mexican intercultural educational models.

KEYWORDS: Language policy, Intercultural education, Indigenous language, Higher education program, Higher Education.

\section{INTRODUCCIÓN}

Desde 2015 la Universidad Veracruzana Intercultural (UVI), entidad académica de la Universidad Veracruzana (UV), ha trabajado en ampliar su oferta educativa de grado y posgrado. Hasta entonces solo impartía una licenciatura -Gestión Intercultural para el Desarrollo-, pero su propósito es ampliarla, 
introduciendo el enfoque intercultural en los estudios universitarios y atendiendo necesidades educativas y sociales detectadas durante su existencia, desde 2004.

Como resultado de varios diagnósticos (Areanorling, 2015, 2016a, 2016b, 2016c), se diseñó un programa de posgrado que contara con una titulación con pertinencia social, cultural y lingüística a las características de la población a la que atiende la UV, teniendo en cuenta que el $8 \%$ de la población juvenil del estado de Veracruz es hablante de una lengua nacional que no es el español, como parte de la Nueva Oferta Educativa (NOE) de la UV.

La Maestría en Lengua y Cultura Nahua (MLCN), como oferta de titulación, representa ya como iniciativa y puesta en acción un modelo de responsabilidad social y un referente para la erradicación de prejuicios y prácticas discriminatorias que en el sistema educativo contribuyen a la reducción y destrucción de la riqueza cultural de México y a la vulneración de los derechos lingüísticos de su población reconocidos en la Ley General de Derechos Lingüísticos de los Pueblos Indígenas (LGDLPI), publicada en el Diario Oficial de la Federación el 13 de marzo de 2003 (DOF, 2018).

Se procuraba por tanto generar un diseño de plan de estudios para una titulación académica adecuada a las necesidades y demandas de profesionistas hablantes de una LIN, lo cual implica establecer un modelo de buenas prácticas que sirva de estímulo y experiencia piloto para que cualquier entidad de educación superior, pública o privada, que vea la viabilidad, oportunidad y pertinencia de reformar y dotarse de programas académicos con pertinencia cultural y lingüística.

Dado que no existían experiencias previas en México, de por sí se dibujaba como un proyecto pionero cuya simple enunciación tendría un efecto ejemplar y un aprendizaje metodológico el cual requeriría seguir una cuidada planificación. Esto garantizaría un modelo coherente, viable y adaptado.

\section{ANTECEDENTES}

Para entender la relevancia e importancia de esta propuesta dentro del marco social e histórico que caracteriza el sistema educativo mexicano, conviene hacer una breve revisión histórica donde se demuestra su pertinencia social y urgencia.

Desde que a comienzos del siglo XIX se independizara el país, el estado mexicano concibió como un elemento para dotar de unidad e identidad nacional la generalización de la lengua española. Se impulsó un ideario castellanizador el cual no logró realmente cristalizarse hasta mediados del siglo XIX con el triunfo de las políticas liberales. La creación de un sistema educativo general y obligatorio sería la condición para dotar al estado de la estructura necesaria para implantar el español como lengua a usarse por toda la ciudadanía mexicana. Es cierto que hubo posturas que propugnaron por una educación adaptada a las condiciones culturales e idiomáticas de las regiones con población indígena, e incluso por incorporar estas lenguas en los planes de estudio de la educación general y bachillerato, pero no lograron prosperar (Heath, 1992).

Así, durante la dictadura liberal del Porfiriato (1876-1911) se impulsó un modelo educativo que reivindicaba un español mexicanizado, y su difusión entre la población mexicana no hispanohablante. Así se interiorizó un imaginario en el cual las lenguas indígenas no tenían cabida ni presencia en la educación al no ser consideradas como lenguas de conocimiento ni darles el estatus como tal, pues se consideraban una amenaza para la unidad del país (Barriga, 2010). Tras la Revolución Mexicana (1910-1920) se creó un Sistema de Educación Pública en 1921 y un Subsistema de Educación Indígena que en la década de 1940 implantó un sistema de educación bilingüe que, a pesar de su indigenismo y bilingüismo, no renunciaba al afán castellanizador, pues las lenguas indígenas solo actuaban como lenguas de comunicación en los primeros años de escolaridad, sin ser parte ni de la instrucción ni de los contenidos centrales. Además, esta educación bilingüe se limitaba a los niveles iniciales y básicos (Dietz y Mateos, 2011).

Para 1978 se creó la Dirección General de Educación Indígena y se trató de apostar por modelos más integracionistas y menos asimilacionistas, respetando y valorando la diversidad cultural y lingüística. Así se 
pasó de un sistema bilingüe bicultural a un modelo intercultural en la década de 1990 (Bertely, 1998), y para 2001 se creó la Coordinación General de Educación Intercultural y Bilingüe y en 2004 las universidades interculturales con este mismo enfoque.

\section{Marco té́rico}

Lo que se evidenció con la creación de las universidades interculturales y con la incorporación de la UVI a la UV en 2007 fue que la oferta educativa universitaria seguía reproduciendo esquemas y comportamientos segregativos y discriminadores que invisibilizaban las posibilidades de adecuar, cubrir y ampliar la oferta formativa, incluso en espacios y contenidos donde no tenía justificación esta exclusión. Esta situación es una consecuencia de lo que personas autoras han señalado como un esencialismo simplista de la puesta en práctica del diálogo intercultural, la carencia de un lenguaje común y la incoherencia entre conocimientos (Dietz y Mateos, 2011) y, por tanto, la posibilidad y capacidad de generar una oferta educativa situada y relacional en un ámbito que reproduce la marginalidad sistémica hacia lo subalterno, a pesar de su valor, vitalidad y emergencia (Olivé, 2013). Esto contrasta con toda una necesidad sentida en América Latina, donde las características plurilingües y multiétnicas de la población acaban develando la paradoja de un sistema educativo que no se corresponde con sus realidades sociales, culturales, lingüísticas y profesionales (Mato, 2008) y, como bien señala el pedagogo quechua Leonel Cerruto, se expresa en el anhelo "de que el sistema educativo superior sea en nuestras propias lenguas" (citado en Saavedra, 2008, p. 124).

Así, se perciben cuatro situaciones que dibujan un nicho de oportunidad:

- No existe en la universidad mexicana un espacio, contenido o programa que permita el desarrollo de las lenguas indígenas nacionales (LIN) como lenguas de transmisión, generación y aplicación del conocimiento.

- No se reconoce ni favorece la competencia comunicativa en LIN en la plantilla docente universitaria (selección, promoción, especialización), no siendo aprovechada o potenciada para la puesta en marcha de programas con enfoque intercultural o pertinencia social.

- No se reconocen, amparan o garantizan los derechos lingüísticos del estudiantado mexicano en su relación con la administración escolar o en su accesibilidad y participación en la actividad académica universitaria.

- No se fomenta la producción científica y académica en las LIN, sea como producción original o traducción.

Estas situaciones reflejan la marginalidad a la que se ha sometido a parte de la población mexicana para impedir su participación en el desarrollo de prácticas letradas y, por tanto, de su literacidad y textualidad en campos especializados del conocimiento, desde sus planteamientos epistemológicos y a través de sus sistemas lingüísticos. Esto amplía el marco general desde el cual plantear la necesidad de una capacitación intelectual y profesional en el campo de la comunicación oral y escrita. Así recuerda Hernández (2013) que:

Un reto al que nos enfrentamos todos los actores que conformamos la vida académica en la educación superior es lograr nuestra participación en actividades comunicativas que favorezcan la integración de comunidades académicas mediante prácticas de lectura y escritura que respondan a las múltiples necesidades y situaciones cotidianas escolares. También el reto es lograr que las prácticas de lectura y redacción no se limiten al desarrollo descontextualizado de habilidades lingüísticas, ni a una socialización académica que sirva simplemente para responder a los requerimientos inmediatos para acreditar asignaturas. La conformación significativa de comunidades y prácticas académicas para la construcción de conocimientos, identidades y significados se puede lograr cuando, además de los docentes y los alumnos, las autoridades y la institución participan en la conformación retroalimentada de propuestas de literacidad en la vida universitaria (p. 17). 
Obviamente, estas situaciones son resultado de un sistema educativo superior que reproduce los prejuicios lingüísticos interiorizados durante siglos, hasta el punto de ocultar la propia viabilidad de una oferta educativa con pertinencia lingüística:

- Existe una matrícula de alumnado originario de comunidades indígenas y hablante de LIN.

- Ha habido un incremento de estudiantes indígenas que cursan programas de posgrado.

- Existe un profesorado que es hablante de LIN.

- Existen políticas educativas que desde las instituciones promueven la introducción del enfoque intercultural en la educación superior al margen de que las universidades sean interculturales o no.

Estas posibilidades de dotar de programas educativos con pertinencia social fueron la base inicial para plantear un diagnóstico que de modo empírico confirmara la viabilidad y oportunidad de generar una propuesta. Se hizo un estudio cuyas conclusiones (Areanorling, 2016a, 2016b) confirmaron estos aspectos y apuntaron como deseable un programa de posgrado (maestría), de carácter profesionalizante y presencial, para nahuahablantes y con contenidos especializados, cuyo diseño se ajustara a la normativa y requisitos establecidos por la Dirección General de la Unidad de Estudios de Posgrado de la UV (DGP).

\section{Metodología Y ACCiones deSARrolladAS}

La planificación de una NOE en náhuatl se diseñó como un proceso abierto que se nutría de aportaciones interdisciplinares de profesionistas nahuas nativos, estudiosos y aprendices. Se siguieron tres etapas:

\section{Etapa 1: Estudio diagnóstico}

Este diseño lo iniciamos en enero de 2015 con un diagnóstico que permitiera observar y dialogar para conocer con certeza las problemáticas, necesidades y/o demandas sentidas por una comunidad, una escuela, una institución o un grupo de personas que desean o les interesa resolver una situación y/o generar nuevos cambios en su entorno social, político, educativo, entre otros.

Se hicieron entrevistas estructuradas a través de cuestionario a personas licenciadas y profesionales, empleados y empleadores del sector público predominantemente, para identificar necesidades y demandas formativas que permitieran establecer las características académicas de la NOE. Se hizo un sondeo y estudio exploratorio que cubrió cinco regiones del estado de Veracruz (Huasteca, Totonacapan, Grandes Montañas, Las Selvas y Xalapa), donde se ubica población que potencialmente requeriría esta NOE y donde se dispone de recursos para ofertarla.

Previamente esta NOE se había formulado a nivel de licenciatura para la atención de lenguas originarias. No obstante, esta propuesta no trascendió porque el equipo base determinó como más viable y efectiva una NOE que atendiera a una lengua en particular, el náhuatl, siendo conveniente una titulación de maestría por ser bastante demandada en el mercado laboral, ser mejor valorada por los futuros aspirantes por su mayor rentabilidad en la búsqueda y promoción de empleo en el sector educativo o a la administración pública, y cubrir un espacio poco atendido por la universidades interculturales o no estar dotado de una oferta seria y formal. Así, el equipo base (EB), en su conjunto y como agente curricular, cuestionaba las improvisaciones y simulaciones que se hacían en distintos programas educativos ofrecidos por instituciones de nivel superior en México.

Las conclusiones se publicaron en varios reportes (Areanorling, 2015, 2016a, 2016b, 2016c) determinando que la NOE más conveniente sería una maestría en náhuatl en modalidad profesionalizante y presencial, la cual fortaleciera competencias en conocimientos y habilidades en lengua, cultura e historia nahua, además de técnicas y métodos de investigación y trabajo aplicados a las telecomunicaciones, la 
educación, la traducción e interpretación, la mediación y promoción, así como la producción y edición de textos. Esto es, fortalecer competencias instrumentales, comunicativas y lingüísticas para el buen desempeño de su profesión en campos tradicionales como la educación bilingüe y en otros emergentes como la interpretación y traducción, la mediación intercultural, la gestión terminológica, la enseñanza como segunda lengua (L2 ${ }^{[1]}$, la producción y edición de textos impresos y audiovisuales en sectores como educación, salud, procuración de justicia e industrias culturales.

Esta etapa diagnóstica fue un primer paso para el trabajo de planificación del náhuatl como lengua vehicular y de trabajo académico y profesional, en tanto que hubo una suma de esfuerzos a unísono, voces diversas en náhuatl y español participando en el diálogo intercultural, cuyos discursos y actitudes de distintos participantes sostuvieron su factibilidad. Ahora bien, como parte de los resultados de esta primera fase destacaron cuatro aspectos (Tabla 1) que hacen viable la oferta educativa (Bernal y Figueroa, 2016):

TABLA 1

Tipos de pertinencia que hacen viable la oferta educativa

\begin{tabular}{llll}
\hline Laboral & Social & Académica & Lingüística \\
\hline Necesidad & Necesidad de & Necesidad de una & Necesidad de \\
urgente de & atender a una & formación superior & fortalecer el uso \\
profesionistas & población en & impartida en lengua & de la lengua \\
con & Situación de & náhuatl para el & náhuatl a nivel \\
competencias & marginación, & desarrollo de & oral, escrito y \\
comunicativas & discriminación y & actividades & mediante las \\
en lengua & desatención, para & especializadas, que & nuevas \\
náhuatl por & cumplir con la & permita su & tecnologias de la \\
parte de & LGDLPI, y de & actualización y & información \\
empleadores. & equidad en una & activación como & para la \\
& sociedad & lengua de & transmisión y \\
& pluricultural y & pensamiento y & generación de \\
& multilingüe. & creación. & conocimiento y \\
& & & estudio de la \\
& & & lengua. \\
\hline
\end{tabular}

Fuente: Elaboración propia

Lo que revela la Tabla 1, motiva a seguir discutiendo y analizando el reto de atender las necesidades en estos cuatro aspectos. Se observa que hay factores sociales que han estado en contra del mantenimiento y actualización de la lengua náhuatl, dado que no se han revertido la marginación y discriminación de sus hablantes. Si se logran mejorar las condiciones académicas y se apuesta por atender esta lengua en espacios como la universidad, esta funcionará como catalizador para posicionar al náhuatl como lengua de prestigio, y garantizará que sus personas egresadas ocupen espacios laborales donde se necesite de su intervención profesional. En este sentido, la sociedad, al ver estos hechos inéditos, podrá otorgar un estatus distinto a su lengua, como lo que actualmente está beneficiando a los egresados de la UVI, hablantes de LIN (Figueroa, Alarcón, Bernal y Hernández, 2014).

\section{Etapa 2: Diseño curricular}

Estas conclusiones del diagnóstico permitieron orientar el diseño curricular. De marzo de 2016 a diciembre de 2017 el equipo diseñador estuvo trabajando en la propuesta del Programa de la Maestría en Lengua y Cultura Nahua.

Ya se ha advertido la relevancia de contar previamente con un diagnóstico para actuar con precisión al momento de plantear un tipo de oferta formativa. Cuando se diseña un programa educativo, se piensa primeramente en los recursos humanos con los que cuenta la propia institución formadora y otras 
instituciones de educación superior que podrían impartir los cursos, con miras a iniciar y sostener un currículum acorde con su modelo educativo. Para ello participaron personas expertas dispuestas a construir la propuesta a partir de sus experiencias, compartiendo en el diálogo intercultural los conocimientos desde los campos disciplinares donde se ha sido formado y actuado consecuentemente en el mercado laboral. Igualmente se sucedieron reuniones con los responsables de la DGP para la adecuación de las propuestas a los formatos, parámetros y condiciones que establece el Reglamento General de Estudios de Posgrado 2010.

El currículum que se propuso es intercultural, pues se pone en diálogo los saberes disciplinares de los sujetos para sentar bases en torno al tema central de la Maestría. Por citar un ejemplo, el título desde el náhuatl originó una discusión amplia entre las personas expertas nativohablantes y estudiosas de esta lengua, pues debía reflejar el contenido y fin de la titulación. Las primeras propuestas de nombre permitieron, al equipo base, reflexionar sobre el carácter de la maestría, dado que fue un primer ejercicio de lingüística aplicada y neología. Estos nombres se resumen en una gran variabilidad de enunciados (Tabla 2) que contribuyera a visibilizar los contenidos del programa y a lograr en el nombre de la titulación una significatividad, claridad y representatividad, tanto en la recepción del futuro estudiante como en la especificación de una NOE.

TABLA 2

Propuestas de denominación de la NOE

\author{
Weyi Tlamachtilli \\ Maestria Mastiliah \\ Mastriah Maestriah
Nawatlahtolli iwan Nemilistli
Tonemilis iwan Totlahtol \\ Ika Ipan Tonemilis iwan Tonawatlahtol
}

\title{
Fuente: Elaboración propia
}

Dichas propuestas fueron enviadas a diversas personas expertas nahuahablantes que no estuvieran involucradas en este proceso, al mismo tiempo, que cada uno de los miembros del equipo base argumentó su propuesta:

[...] la propuesta de nombre que más me hace sentido es "Weyi tlamachtilli ipan tonemilis iwan tonawatlahtol", que traduzco como "gran enseñanza en cultura y lengua náhuatl” (J. A. Flores Martínez, comunicación personal, 15 de marzo de 2016).

[...] la propuesta de nombre que más me hace sentido es "Weyi tlamachtilli ipan tonemilis iwan tonawatlahtol", que traduzco como "gran enseñanza en cultura y lengua náhuatl" (J. A. Flores Martínez, comunicación personal, 15 de marzo de 2016).

La primera (Tonemilis iwan Tonawatlahtol) y la tercera (Tonemilis iwan Totlahtol) corresponden perfectamente a la estructura morfológica y sintáctica del náhuatl. Ambas significan (pragmáticamente) exactamente lo mismo. La ventaja de la tercera es que es más breve. Tonemilis (nuestra vida) se entiende, en este contexto por nuestra forma de vida). Siempre es preferible una forma breve (economía de lenguaje), por lo que la tercera (Tonemilis iwan Totlahtol) es preferible (A. Hasler Hangert, comunicación personal, 15 de marzo de 2016).

Les recuerdo lo que nos dijo el colega Jaime sobre la crítica al concepto cultura y su acercamiento a forma de vida. Por otra parte, creo que el concepto nemilistli le da un enfoque biocultural al concepto de cultura que trasciende lo humano y lo histórico y lo lleva a su naturaleza orgánica y activa, personal y colectiva, nómada y sedentaria (M. Figueroa, comunicación personal, 16 de marzo de 2016).

[...] les comento que entre algunos estudiantes de la UVI Montañas, la palabra toltecayotl se está generalizando como "cultura" en general (ni siquiera necesariamente como cultura náhuatl, y también la he visto utilizada así en ocasiones fuera de la UVI acá en la región. Aunque es cierto que no se trata de una palabra transmitida por generaciones, sino una palabra introducida por los intelectuales nahuas contemporáneos, pero que, en un nivel muy incipiente, ya está siendo usada por más nahuahablantes [...] Yo voto porque el nombre quede con tonemilis, y que esta discusión sobre la toltekayotl se lleve a otro espacio (C. O. Sandoval Arenas, comunicación personal, 16 de marzo de 2016).

Te cuento que el nombre que me hace más [sic.] conexión es: Weyi tlamachtilli ipan nawatlahtolli iwan nemilis (A. Hernández, comunicación personal, 29-3-2016) 
Finalmente, el consenso fue Maestriah ipan Totlahtol iwan Tonemilis por las connotaciones y carga semántica que tienen los términos como reivindicación de una visión propia del conocimiento.

El currículum se afianzó durante su construcción mediante la recontextualización e internalización de las propuestas macrodiscursivas y macropolíticas (Díaz, 2007) de las políticas lingüísticas y educativas iniciada por la CGEIB y por el Instituto Nacional de Lenguas Indígenas, además de la misma UVI. Los diseñadores debieron establecer las materias-contenidos de acuerdo con tres Líneas de Generación y Aplicación del Conocimiento (LGAC): 1) Mediación Lingüística Intercultural, 2) Gestión, Planeación y Revitalización Lingüística, y 3) Enseñanza y Aprendizaje de la Lengua. ${ }^{[2]}$ Estas líneas integran las necesidades y demandas formativas en un perfil profesional multidisciplinar, complementario y especializado, bien valorado en la oferta de empleo público y de trabajo comunitario.

De este modo, los cursos fueron organizados según una malla curricular que establecería los contenidos troncales o comunes, y optativos según la orientación disciplinar y de intervención de la formación. En esto hay que señalar que, como formación profesionalizante, el objetivo de la Maestría es que el estudiantado desarrolle proyectos de investigación-intervención en sus ámbitos profesionales. Los cursos fueron consensuados de la siguiente manera (Tabla 3):

TABLA 3

Cursos de la Maestría en Lengua y Cultura Nahua.

\begin{tabular}{|c|c|c|c|}
\hline \multicolumn{4}{|c|}{ Cursos obligatorios: 14} \\
\hline \multicolumn{2}{|c|}{ Gramática del náhuatl } & \multicolumn{2}{|c|}{ Comunicación interdialectal } \\
\hline \multicolumn{2}{|c|}{ Lectura y redacción especializada } & \multicolumn{2}{|c|}{ Retórica y oratoria } \\
\hline \multicolumn{2}{|c|}{ Historia y cultura nahua } & \multicolumn{2}{|c|}{$\begin{array}{l}\text { Proyecto de intervención I: diseño y } \\
\text { planeación }\end{array}$} \\
\hline \multicolumn{2}{|l|}{ Lengua y sociedad } & \multicolumn{2}{|c|}{$\begin{array}{l}\text { Proyecto de intervención II: } \\
\text { implementación }\end{array}$} \\
\hline \multicolumn{2}{|c|}{ Gestión del conocimiento nahua } & \multirow{2}{*}{\multicolumn{2}{|c|}{$\begin{array}{l}\text { Proyecto de intervención III: análisis } \\
\text { de resultados y evaluación }\end{array}$}} \\
\hline \multicolumn{2}{|l|}{ Políticas lingüísticas } & & \\
\hline $\begin{array}{l}\text { Optativa I . } \\
\text { Traducción . } \\
\text { Iniciativas y acciones } \\
\text { para la revitalización } \\
\text { del náhuatl } \\
\text { Enseñanza del } \\
\text { náhuatl como L2 }\end{array}$ & \multicolumn{2}{|c|}{$\begin{array}{l}\text { Optativa II . } \\
\text { Interpretación . } \\
\text { Gestión lexicográfica } \\
\text { Diseño de materiales }\end{array}$} & $\begin{array}{l}\text { Optativa III · Mediación } \\
\text { comunicativa · } \\
\text { Documentación lingüística } \\
\text { Enseñanza del náhuatl } \\
\text { como L1 . Comunicación } \\
\text { editorial . Comunicación } \\
\text { publicitaria } \quad \text { Creación } \\
\text { literaria }\end{array}$ \\
\hline
\end{tabular}

Fuente: Elaboración propia.

Se establecieron también aspectos como: 1) los recursos humanos, materiales y de infraestructura disponibles, que determinan por ejemplo el centro de ubicación de la carrera; 2) el perfil del alumnado y requisitos de ingreso que define al potencial estudiante como un profesional nahuahablante, nacional o extranjero, que quiera desarrollar su carrera profesional en comunidades nahuas o atender necesidades asociadas; 3) un perfil y requisitos de permanencia, egreso y titulación que establece el nivel de desempeño requerido para la superación del programa; 4) el perfil académico que establece las competencias profesionales y titulación requerida para ser miembro del Núcleo Académico Básico (NAB) o docentes colaboradores; 5) el diseño curricular propiamente dicho, la duración de los estudios que se estableció en dos años, con un total de 1.115 horas y 119 créditos, y 6) la descripción del reconocimiento académico que establece la expedición 
de un certificado bilingüe con la solicitud de registro de la titulación como Maestriah ipan Totlabtol iwan Tonemilis.

\section{Etapa 3: Revisión y validación institucional}

Durante esta etapa debieron hacerse ajustes al plan de estudios para adecuarlo a parámetros curriculares, distribuir el horario y carga docente, y definir criterios que después servirán para abrir la convocatoria y para elaborar la autoevaluación del programa, además de facilitar la conformación del NAB, estableciéndolos de acuerdo con criterios de calidad según parámetros establecidos por la DGP y estar en posibilidad de que este programa ingrese a Consejo Nacional de Ciencia y Tecnología ${ }^{[3]}$ toda vez que se evalúe a la primera generación.

Se realizaron sesiones de presentación y revisión de la propuesta del plan de estudios y documentación, con el objetivo de afinar los detalles. Igualmente, estos ajustes y correcciones permiten que el programa sea aprobado para su implementación.

Sin embargo, es importante señalar que estas reuniones también sirvieron para que la DGP se hiciera eco de aspectos y procedimientos que desde la propia Universidad impiden la generación de programas educativos inclusivos y la falta de reconocimiento efectivo y administrativo de la diversidad lingüística propia de la comunidad universitaria.

\section{Organización}

Un diseño de estas características requirió de varios grupos de trabajo.

En la Etapa 1 se conformó el equipo base con cinco docentes de la UVI, un miembro investigador del Instituto de Investigaciones en Educación (IIE) de la UV y un miembro investigador del Centro de Investigaciones y Estudios Superiores en Antropología Social (CIESAS-Golfo). En el Gráfico 1 se observa el perfil lingüístico de cada uno de los miembros:

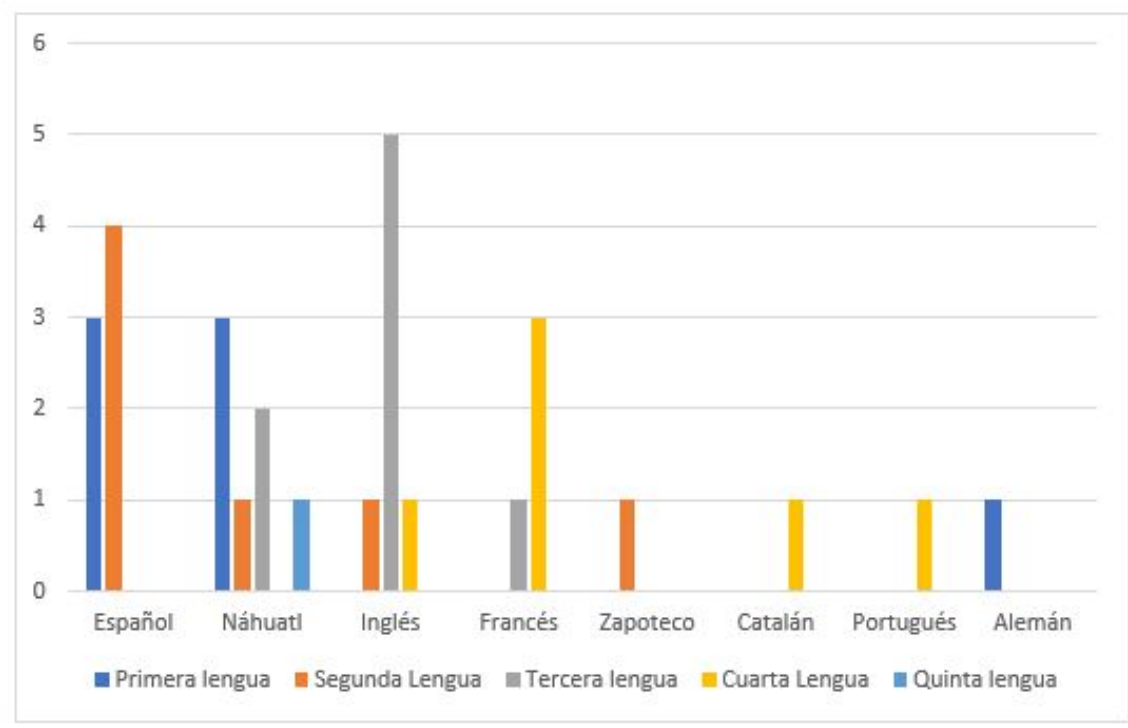

\section{GRÁFICO 1}

Adquisición, conocimiento y estudio de lenguas del equipo base

Fuente: Elaboración propia 
Posteriormente, se sumarían puntualmente especialistas y expertos, quienes analizarían y establecerían las características de esta NOE a partir de un estudio de antecedentes y diagnóstico de necesidades. Este otro grupo de personas expertas que opinaban sobre los resultados obtenidos en el sondeo y participaban en el diagnóstico, conformaban el equipo de colaboradores. Participó personal académico de las cuatro sedes regionales de la UVI, de la Casa-UVI y del IIE de la UV, estudiantes de la UVI y de la licenciatura de Antropología Lingüística en servicio social.

A estos hay que sumar obviamente a los grupos de colaboración conformados por egresados, profesionistas y especialistas que accedieron a ser entrevistados, y a los representantes de entidades empleadoras:

1. 67 profesionistas hablantes de LIN de Veracruz con especial interés en personas egresadas de la Licenciatura en Gestión Intercultural para el Desarrollo,

2. 15 entidades empleadoras y trabajadores independientes que para el cumplimiento de sus funciones laborales requieren de la aplicación de competencias lingüísticas y comunicativas en lenguas indígenas nacionales, $\mathrm{y}$

3. 14 especialistas en el campo de la lingüística y la educación de instituciones veracruzanas y de otros estados de México.

El equipo implementó el enfoque interactoral que promueve la UVI en la investigación vinculada, en cuanto a considerar la participación de diferentes actores que opinaron desde sus conocimientos y experiencias, lo cual fortaleció la apuesta de la política lingüística explícita que hace la UV. Además, se logró un amplio reconocimiento de las regiones, ámbitos y sectores en los que esta oferta tendría impacto (Areanorling, 2016a, 2016b).

En la Etapa 2 se incorporó un equipo de diseñadores, es decir, de responsables de diseñar los contenidos, dinámicas y procedimientos de evaluación de los cursos, componiéndose con miembros del equipo base y de especialistas. El equipo base internamente, pero también con el equipo de diseñadores generaron discusiones, análisis y reflexiones a partir de temas en común, que orientaron y retroalimentaron la propuesta para cada curso, mismas que permitieron construir los consensos para sumar y clarificar los contenidos, las metodologías, las formas de evaluación y los referentes que cobijan la apuesta como visión, misión y objetivos del programa en su conjunto.

También hubo reuniones puntuales con los representantes de la DGP para ajustar las características de los programas de estos cursos con los requisitos de los programas de posgrado en atención al carácter profesionalizante y la distribución de créditos teóricos y prácticos y de horas áulicas con personal docente o sin este, y no áulicas.

En la Etapa 3 se acentuaron las reuniones del equipo base con la DGP para revisar el conjunto del plan de estudios de acuerdo con los formatos y criterios establecidos por reglamento para su aprobación por el Consejo Consultivo de Posgrado, el Consejo de Área y finalmente por el Consejo General Universitario.

Este proyecto y la actividad generada fue auspiciada por la Dirección de la UVI quien propuso como coordinadora de este trabajo al Área de Normalización Lingüística y nombró una comisión integrada principalmente por académicos de la propia UVI, a la que se sumaron miembros del Cuerpo Académico de Estudios Interculturales del IIE de la UV.

Esta conformación logró operar la Fase Diagnóstica y obtener resultados que permitieron reconfigurar el equipo de trabajo con perfiles específicos y especializados para el diseño curricular de la MLCN, como se ha mencionado. Se contó también con el apoyo expreso de la Secretaría Académica de la UV y de la DGP. En esto fue importante contar con recursos y permisos para que el personal académico de la UVI pudieran llevar a cabo sus reuniones y gestiones sin perjuicio de sus compromisos laborales. 


\section{RESULTADOS Y PERSPECTIVAS}

La propuesta de diseño curricular ha generado expectativas desde que los resultados de la Fase Diagnóstica fueran presentados en distintos eventos académicos como Seminarios, Foros, en reuniones de trabajo para compartir la experiencia con otras universidades interculturales y especialmente se llegó a un público para el que se pensó el programa, en el Segundo Encuentro de Hablantes de Lengua Náhuatl (Bernal y Antonio, 2016) donde se recibieron comentarios, críticas, reflexiones y propuestas. Estas participaciones contribuyeron en el cumplimiento del diseño de un currículum intercultural cuyo plazo de entrega era en diciembre de 2017. Así que esta propuesta es, en los términos de Aguado (2003), en cuanto al currículo, señala que "la propuesta cultural de la escuela es la formalización de la cultura escolar, la que el currículo transmite y legitima" (p. 114).

Tras el diseño del programa queda la continuación de este proyecto de NOE con la puesta en marcha de este, lo cual requiere de la realización planificada de otras actividades, ya con los miembros del NAB y las autoridades directamente responsables del programa. A tal respecto, se prevé un cambio en la articulación del equipo base, ya como colegio de docentes en la modalidad de tequio académico (trabajo colectivo). Dada la fuerte exigencia a nivel pedagógico que un proyecto así requiere, se necesita contar con cursos y/o talleres que fortalezcan la formación como docente intercultural en nivel de posgrado y permita formalizar una didáctica específica, activando y desarrollando las funciones comunicativas del náhuatl en un lenguaje académico y adecuar los saberes y cosmovisión nahua en una epistemología articulable en disciplinas de conocimiento y habilidades y técnicas profesionales. Esto supondrá ir facilitando la producción académica en náhuatl aplicada a las necesidades operativas de las LGAC. Considerando lo anterior, el tequio académico tiene proyectados cuatro aspectos que serán trabajados de manera creativa y crítica en función de las necesidades que el propio programa vaya revelando (Tabla 4).

TABLA 4

Aspectos a desarrollar para puesta en marcha del programa

\begin{tabular}{|c|c|}
\hline Diseño de instrumentos & $\begin{array}{l}\text { 2) Elaboración y compilación de } \\
\text { materiales }\end{array}$ \\
\hline $\begin{array}{l}\text { *Guion de entrevista oral en lengua } \\
\text { nahua para conocer los intereses de } \\
\text { trabajo de los aspirantes *Diseño de } \\
\text { examen de competencias comunicativas } \\
\text { en lengua nahua Convocatoria por parte } \\
\text { del equipo base *Agenda de fechas para } \\
\text { la aplicación y evaluación del examen en } \\
\text { lengua nahua *Elaboración y diseño de la } \\
\text { convocatoria de la Maestría }\end{array}$ & $\begin{array}{l}\text { "Antologias "Manuales prácticos } \\
\text { "Presentación de power point }\end{array}$ \\
\hline 3) Traducción de textos & $\begin{array}{l}\text { 4) Formación y autoformación } \\
\text { colectiva }\end{array}$ \\
\hline $\begin{array}{l}\text { *Bibliografía básica *Audiovisuales } \\
\text { *Publicaciones del equipo base (artículos } \\
\text { de investigación y de difusión; notas } \\
\text { periodísticas, entre otros) }\end{array}$ & $\begin{array}{l}\text { *Cursos disciplinarios y lo pedagógicos } \\
\text { *Talleres disciplinarios y lo } \\
\text { pedagógicos *Seminario para la } \\
\text { generación de un lenguaje académico } \\
\text { básico común de entre el personal } \\
\text { docente de la MLCN y los aspirantes } \\
\text { seleccionados }\end{array}$ \\
\hline
\end{tabular}

Fuente: Elaboración propia

El rubro de Diseño de instrumentos sentará un precedente procedimental para la selección de aspirantes competentes a ingresar a la Maestría. 
En cuanto a la Elaboración y compilación de materiales, es un trabajo que se ha pensado como: a) compilar textos escritos en y desde el náhuatl, generarlos o integrar textos en español o en otra lengua si se trata de clásicos, cuya comprensión el profesorado deberá facilitar en náhuatl y español; b) elaborar manuales que faciliten el proceso de enseñanza-aprendizaje de una forma más clara y lúdica para la comprensión y el análisis del náhuatl; y c) preparar las clases en náhuatl con el apoyo de paquetería informática básica para incentivar la discusión, el análisis y las reflexión en las clases.

Sobre el aspecto de Traducción de textos, se debe partir de las fuentes originales, sean en español o en lenguas extranjeras para traducirse al náhuatl. La traducción seguirá siendo un ejercicio del quehacer del equipo base antes y durante la implementación del programa. El cuerpo de docentes, junto con los maestrantes nahuahablantes, estará generando un lenguaje común, académico e interdialectal en lengua nahua.

En lo que respecta al aspecto Autoformación y formación colectiva, será imprescindible que el personal docente reciba capacitación colectiva previa a la impartición de los cursos, con el fin de que caminen en una misma dirección y hacia una misma meta colectiva institucional y comunitaria, y constituya un sólido apoyo para la autoformación para cada uno de los cursos.

En este punto se iniciaría la vinculación interinstitucional prevista con universidades nacionales y extranjeras a partir de convenios académicos vigentes con entidades como la Confederation College of Applied Arts and Technology (Canadá), la Universidad de Leyden (Holanda), la Universidad de Chicago (EE.UU.), y la Universidad Nacional Autónoma de México, al contar con estudios, líneas de investigación y perfiles profesionales afines con los propósitos del Programa. También se prevén futuras colaboraciones y convenios con la Universidad Iberoamericana, Universidad Autónoma de San Luis Potosí, Universidad de Guadalajara, Universidad de Varsovia, Universidad de Arizona, Universidad de Yale, Universidad de Texas, Universidad de Utah, Universidad de Oregón y Universidad de Michigan, por contar con programas y proyectos para la formación en lengua y cultura nahua. De este modo se promoverían estancias académicas entre docentes, estudiantes y profesionales.

\section{Aspecto clave y recomendaciones}

Como se ha dicho, proponer y elaborar un programa de estas características no es fácil. Primero existen bastantes prejuicios que consideran de partida que este tipo de iniciativas no son necesarias. Evidentemente, estas apreciaciones no descansan en estudios o diagnósticos, sino en el presupuesto de que el México del siglo XXI es un país monocultural y monolingüe con la única expectativa de implantar un modelo bilingüe español-inglés. Nada más lejos de la realidad.

El hecho es que reconocer la diversidad lingüística, en este caso, del estado veracruzano, representa también el reconocimiento empírico de que es una población universitaria que demanda y necesita una formación. Además, se evidencia que dicha formación debe contribuir a desarrollar sus conocimientos sobre la cultura nahua y sus habilidades comunicativas para realizar un trabajo más efectivo e integrado en sus ámbitos de trabajo y comunidades de pertenencia en campos profesionales hoy -valga la paradoja-poco o nada profesionalizados.

Por tanto, la propuesta y arranque de una oferta educativa en la universidad de este cariz confirma el carácter pluricultural de la nación ya reconocido por el artículo 2 de la Constitución Política de los Estados Unidos Mexicanos. En este sentido, parte del ánimo de avanzar en el proyecto fue demostrar ante las autoridades educativas la viabilidad y factibilidad de programas con pertinencia social, cultural y lingüística, con una demanda y utilidad social tangible y para la cual se requiere de su colaboración.

Por tal razón, es evidente que este tipo de iniciativas requiere de un gran esfuerzo de sensibilización y concienciación, de adecuación de las estructuras y normas, de detección y selección de recursos humanos 
capacitados, de identificación de perfiles profesionales y disciplinares clave, de promoción y apoyo en su puesta en marcha.

Por tanto, la elaboración de un plan de estudios así sirve para desmontar falsos límites e imposibles, y demostrar a otras iniciativas que se están produciendo en estados como Ciudad de México, Puebla, Chiapas, Yucatán, Oaxaca o Tabasco, por citar algunos, y otros países latinoamericanos, que en determinadas LIN se está en condiciones de generar una NOE y cumplir con la función social de la Universidad de atender a las necesidades de desarrollo y participación de la ciudadanía.

\section{Conclusiones}

El diseño curricular de una oferta educativa que desde un enfoque intercultural y una pertinencia social, cultural y lingüística promueva planes de estudio los cuales puedan ser impartidos en una lengua minorizada, requiere no solo de un sustento y apoyo legal, sino sobre todo de un trabajo previo de diagnóstico de necesidades y viabilidad que le dé fundamentos sólidos a la propuesta. Esto supone implicar a todos aquellos agentes que de alguna manera van a refrendar en un sentido funcional, el valor de esta nueva formación y titulación. De este modo la universidad como entidad acreditadora no dota solo de prestigio a la acción de planificación educativa y lingüística, sino que incide en el mismo estatus de profesionistas que manejan de modo profesional una lengua indígena nacional (y por tanto de su comunidad de habla que representan). A esto le sigue una serie de etapas las cuales permiten obtener información válida y validada que oriente el plan de trabajo y permita al equipo encargado hacer la propuesta con las garantías de que se ajuste a los criterios académicos de la oferta universitaria y de que la formación impartida satisfaga las expectativas de profesionistas, comunidad y empleadores. Por eso mismo el diseño de entrevistas para aplicar entre posibles personas interesadas en esta formación profesionalizante y aquellas personas que reconocer la necesidad social y laboral de titulados con un perfil lingüístico adecuado al destinatario de productos y servicios, y las entrevistas enfocadas a expertos para obtener su asesoramiento y corroboración de lo identificado en campo, permite contar con datos que ayudan a la toma de decisiones del equipo base de cara a ofrecerse un programa y plan de estudios bien justificado y vinculado. Para ello también ha sido importante la labor de sensibilización y toma de conciencia de las autoridades universitarias y los ajustes de las normativas universitarias a la Ley General de Derechos Lingüísticos. De este modo se logra una nueva oferta formativa que cambie el carácter aún monolingüístico, monocultural y monológico de la educación superior, distanciado de las características y prácticas cotidianas de la sociedad mexicana.

\section{REFERENCIAS BIBLIOGRÁFICAS}

Aguado, T. (2003). Pedagogía intercultural. España: McGraw-Hill.

Areanorling (Área de Normalización Lingüística). (2015). Reporte del estudio piloto aplicado a empleadores 2015. Xalapa, México: Universidad Veracruzana Intercultural. Recuperado de https://bit.ly/2DXM2tD

Areanorling (Área de Normalización Lingüística). (2016a). Reporte de situación laboral y necesidades formativas de egresados 2015. Xalapa, México: Universidad Veracruzana Intercultural. Recuperado de https://bit.ly/2UL5yz9

Areanorling (Área de Normalización Lingüística). (2016b). Reporte de necesidades laborales y formativas de empleadores 2015. Xalapa, México: Universidad Veracruzana Intercultural. Recuperado de https://bit.ly/2HYQUTy

Areanorling (Área de Normalización Lingüística). (2016c). Análisis de entrevistas a especialistas en Lengua y Educación 2015. Xalapa, México: Universidad Veracruzana Intercultural. Recuperado de https://bit.ly/2SgB4It

Barriga, R. (2010). Una hidra de siete cabezas y más: La enseñanza del español en el siglo XX mexicano. En R. Barriga Villanueva y P. Martín Butragueño (Directores), Historia sociolingüistica de México (pp. 1095-1194). México: El Colegio de México. 
Bernal, D. y Figueroa, M. (Agosto de 2016). Diagnóstico para una oferta educativa en náhuatl: hacia la pertinencia lingüística en la educación superior. En II Seminario de Investigación Universidades Interculturales en México: balance de una década. Ponencia presentada en Universidad Nacional Autónoma de México, México.

Bernal, D. y Antonio, F. (Agosto, 2016). Segundo Encuentro de Hablantes de Lengua Nábuatl en la UVI Las Selvas. Recuperado de https://bit.ly/2DwkWsj

Bertely, M. (1998). Educación indígena del siglo XX en México. En P. Latapí Sarre (Coord.), Un siglo de educación en México (pp. 74 -110). México: CONACULTA, FCE.

Díaz, M. (2007). Prólogo. En A. de Alba, Curriculum-sociedad. El peso de la incertidumbre, la fuerza de la imaginación (pp.25-34). México: UNAM y Plaza y Valdés.

Dietz, G. y Mateos, L. S. (2011). Interculturalidad y educación intercultural en México: un análisis de los discursos nacionales e internacionales en su impacto en los modelos educativos mexicanos. México: SEP, CGEIB.

DOF (Diario Oficial de la Federación). (2018). Ley General de los Pueblos Indigenas de México. Última reforma DOF 20-06-2018. México: Cámara de Diputados del H. Congreso de la Unión. Recuperado de https://bit.ly/2C9 GZpp

Figueroa, M., Alarcón, D., Bernal, D. y Hernández, J. A. (2014). La incorporación de las lenguas indígenas nacionales al desarrollo académico universitario: la experiencia de la Universidad Veracruzana. Revista de la Educación Superior, 43(171), 67-92.

Heath, S. B. (1992). La politica del lenguaje en México. México: INI, CONACULTA.

Hernández, E. (2013). Panorámica sobre literacidad y escritura académica en la educación superior. En H. Muñoz (Ed.), Textualidad y lengua extranjera en el desarrollo académico de estudiantes bilingües en la educación superior (pp.17-48). México: UAM-Itztapalapa.

Mato, D. (Coord.). (2008) Diversidad cultural e interculturalidad en educación superior. Experiencias en América Latina. Caracas: ASCUN, UNESCO. Recuperado de https://bit.ly/2DfZesq

Olivé, L. (2013). Hacia un modelo de sociedades plurales de conocimientos y el pluralismo epistemológico. En S. E. Hernández Loeza, M.I. Ramírez Duque, Y. Manjarrez Martínez y A. Flores Rosas (Coords.), Educación intercultural a nivel superior: reflexiones desde diversas realidades latinoamericanas (pp. 35-50). México: UIEP, UCIRED, UPEL.

Saavedra, J.L. (2008) Universidad Intercultural Indígena Originaria Kawsay (Bolivia). En D. Mato (Coord.), Diversidad cultural e interculturalidad en educación superior. Experiencias en América Latina (pp. 115-124). Caracas: ASCUN, UNESCO.

\section{Notas}

[1] A partir de este punto, se abrevia la lengua primera o primera lengua como L1 y segunda lengua como L2.

[2] Datos considerados en la Presentación del Programa de la Maestriah ipan Totlahtol iwan Tonemilis (Maestría en Lengua y Cultura Nahua) a la Coordinación de Planes y Programas de Posgrado UV el 12 de enero de 2018 por el equipo base de Diseño Curricular de la UVI.

[3] El Consejo Nacional de Ciencia y Tecnología, más conocido como Conacyt es un organismo descentralizado del Gobierno Federal de México cuya función es la promoción y desarrollo de la ciencia y la tecnología, diseñando e implementando políticas a tal fin. Esta institución evalúa y acredita programas de posgrado y apoya a estudiantes para poder cursarlos, en tanto que estén reconocidos como programas de calidad.

1 Esta investigación se realizó en el marco del proyecto "Planificación y políticas del lenguaje en el diseño curricular de los programas educativos de náhuatl y totonaco: el caso de la Universidad Veracruzana Intercultural (No. de Registro DGI: 22211201723) de la Universidad Veracruzana.

2 This research was made into the project "Planificación y políticas del lenguaje en el diseño curricular de los programas educativos de náhuatl y totonaco: el caso de la Universidad Veracruzana Intercultural/Language planning and policies in the design of the nahuatl and totonaco educational programs: the case of the Universidad Veracruzana Intercultural“ (No. de Registro DGI: 22211201723) of the Universidad Veracruzana.

\section{BY-NC-ND}

